Field-induced transformations in the biaxial order of non-tilted phases in a bent-core smectic liquid crystal

This article has been downloaded from IOPscience. Please scroll down to see the full text article.

2010 EPL 9226002

(http://iopscience.iop.org/0295-5075/92/2/26002)

View the table of contents for this issue, or go to the journal homepage for more

Download details:

IP Address: 134.226.252.160

The article was downloaded on 02/03/2011 at 10:23

Please note that terms and conditions apply. 


\title{
Field-induced transformations in the biaxial order of non-tilted phases in a bent-core smectic liquid crystal
}

\author{
Y. P. Panarin ${ }^{1,2}$, Mamatha Nagaraj $^{1}$, J. K. Vij ${ }^{1(a)}$, C. Keith ${ }^{3}$ and C. Tschierske ${ }^{3}$ \\ ${ }^{1}$ Department of Electronic and Electrical Engineering, School of Engineering, Trinity College, University of Dublin \\ Dublin 2, Ireland, EU \\ ${ }^{2}$ School of Electronic $\&$ Communication Engineering, Dublin Institute of Technology - Dublin, Ireland, EU \\ ${ }^{3}$ Institute of Chemistry, Organic Chemistry, Martin-Luther-University Halle-Wittenberg - D-06120 Halle, \\ Germany, EU
}

received 11 August 2010; accepted in final form 27 September 2010

published online 5 November 2010
PACS 61.30.Gd - Orientational order of liquid crystals; electric and magnetic field effects on order

PACS 77.80.-e - Ferroelectricity and antiferroelectricity

PACS 78.20.Fm - Birefringence

\begin{abstract}
The structural and electro-optic investigations of an achiral bent-core molecule in $\mathrm{Sm}_{A} P_{A}$ phase, in which the polar directors in the neighboring layers are arranged antiferroelectrically, show that it undergoes transformation from one biaxial to another biaxial structure via a quasi-stable uniaxial structure on the application of the electric field. The noncontinuous change in biaxiality is explained by an intermediate state in which the secondary directors in the neighboring layers are perpendicular to each other.
\end{abstract}

Copyright (C) EPLA, 2010

Ferroelectricity in tilted smectic phases of chiral molecules predicted on symmetry considerations in 1975 was observed [1] and subsequently extensively investigated. In 1992, Brand et al. [2] suggested another model of ferroelectricity for an orthogonal smectic phase $\left(C_{P}\right)$ formed by non-chiral bent-core (or banana, bow, boomerang) molecules. Such a phase possesses an orthorhombic symmetry $\left(C_{2 v}\right)$, in which one of the three mirror planes is absent and the spontaneous polarization is parallel to the 2 -fold axis. The first experimental evidence of ferroelectricity in non-chiral bent-core smectic LCs was given in 1996 by Niori et al. [3] through measurements of the polarization switching current and of the dielectric permittivity. An important feature of the bent-shaped molecules is their tendency to form tilted smectic phases with macroscopic chirality in the opposite directions [4-6]. Since the first works [1] on banana-shaped molecular systems, a wide variety of different tilted phases such as B1-B8, have been discovered and classified [7-10]. Among these B2 is the SmC-like structure which may form four different structures designated as $\mathrm{Sm}_{S / A} P_{F / A}$, where subscripts $S / A$ refer to synclinic/anticlinic tilt and $F / A$ refer to ferroelectric/antiferroelectric orderings in the neighboring

(a) E-mail: jvij@tcd.ie layers. In fact the first evidence of ferroelectricity in a non-chiral system [3] was found in the B2 phase. Dipolar order has also been observed in non-tilted antiferroelectric bent-core smectic systems $[11,12]$. These are also known as orthogonal $\mathrm{Sm} A$-like phases. The advantage of orthogonal smectic phases as compared to the tilted phases is that problems associated with $\mathrm{Sm} C$-type materials such as a formation of the chevron structures and defects can be avoided. The orthogonal phases of the bent-core materials consist of $\operatorname{Sm} A P_{A}$ and $\operatorname{Sm} A P_{R}$. $\operatorname{Sm} A P_{A}$ was first discovered by Eremin et al. [11] who also proposed that the polar directors in the neighboring layers were packed antiferroelectrically and ferroelectricity was induced by the application of the electric field above a threshold value. The $\operatorname{Sm} A P_{R}$ phase with uniaxial structure was discovered by Pociecha et al. [13], where they postulated a random arrangement of the polar directors among the neighboring layers. Shimbo et al. [14] demonstrated that the biaxiality in the $\operatorname{Sm} A P_{R}$ phase was induced by the electric field and the structure was shown to transform into that of a ferroelectric structure. They also demonstrated this mode for its use in the new LCD technologies of the next generation of displays [15]. It is easier to align materials in $\mathrm{Sm}_{A} P_{R}$ and $\mathrm{Sm}_{A P_{A}}$ similar to the biaxial nematics. In the latter phase, the switching is due to the interaction of the minor director with the electric field, $E$. Since the 


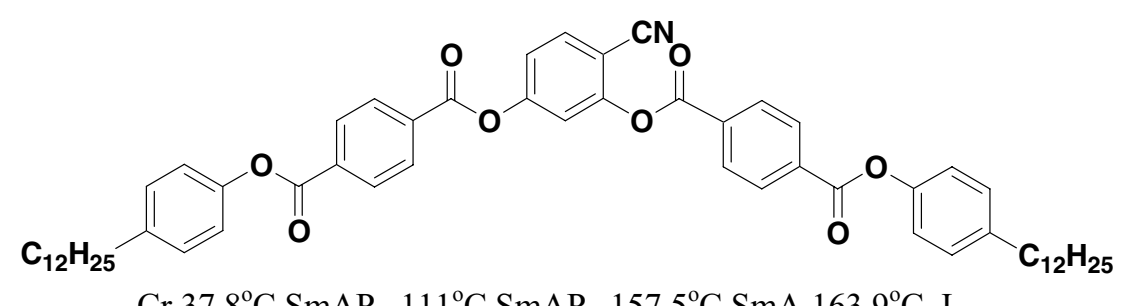
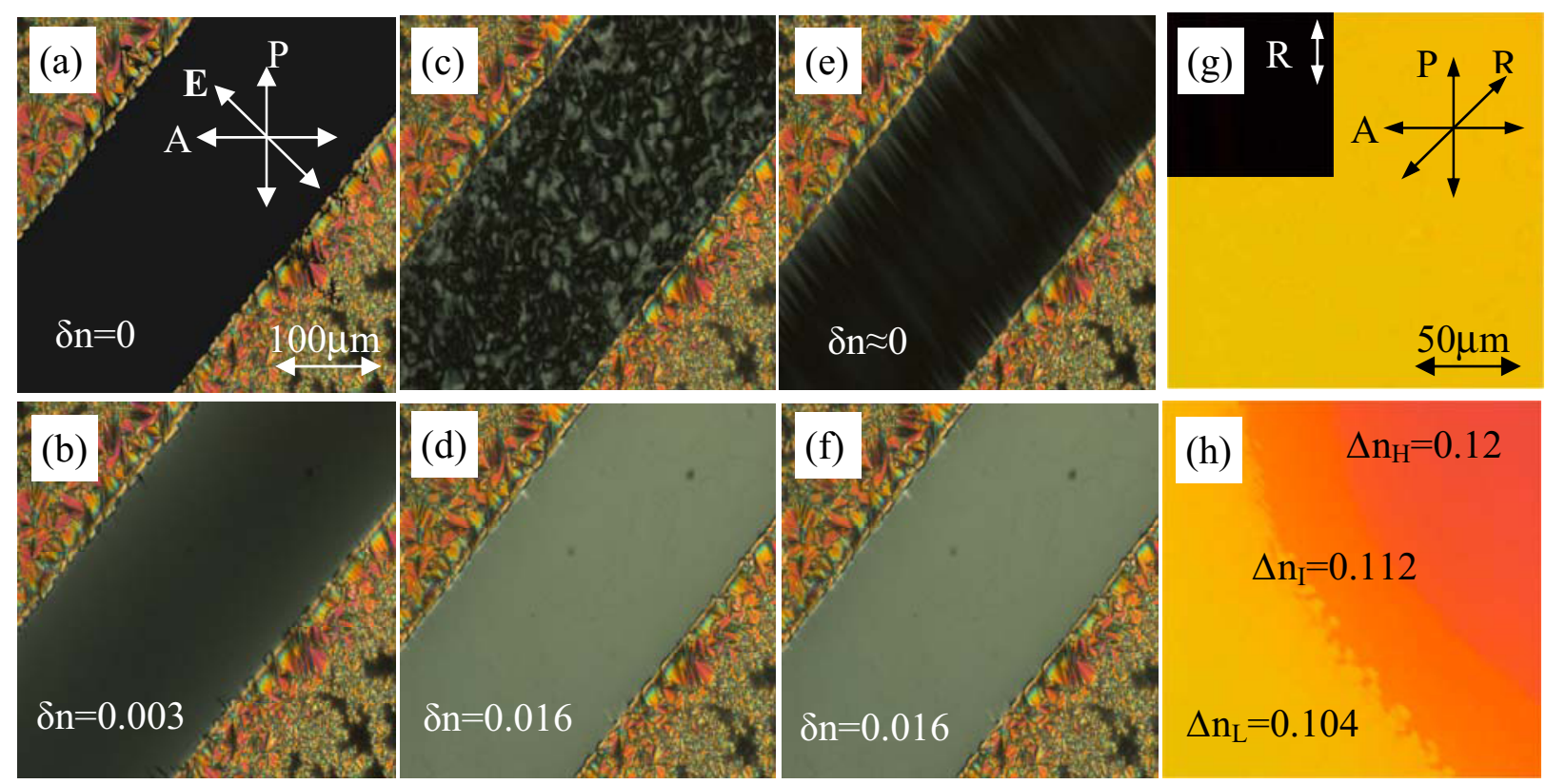

Fig. 1: (Colour on-line) Top: structural formula and the transition temperature obtained on cooling. Textural transformations in $8.7 \mu \mathrm{m}$ homeotropic (a)-(f) and $9.5 \mu \mathrm{m}$ planar cell (g), (h). (a) and (b) are in $\operatorname{Sm} A P_{R}$ phase at $113^{\circ} \mathrm{C}$, without $\boldsymbol{E}$ and with $\boldsymbol{E}=1.4 \mathrm{~V} / \mu \mathrm{m}$ respectively. (c) $-(\mathrm{h})$ are in $\operatorname{Sm} A P_{A}$ phase at $95{ }^{\circ} \mathrm{C}$, where (c) is without $\boldsymbol{E}$, (inset: schlieren texture in a fresh cell), (d) at $0.35 \mathrm{~V} / \mu \mathrm{m}$ (e) at $1.68 \mathrm{~V} / \mu \mathrm{m}$ and (f) at $2.5 \mathrm{~V} / \mu \mathrm{m}(\mathrm{g})$ in $\mathrm{Sm} A P_{A}$ phase at $95^{\circ} \mathrm{C}$ for $0^{\circ}$ (inset) and $45^{\circ}$ angle of the rubbing direction with respect to the polarizer. (h) Three states of molecular alignment resulting in three birefringence values. $\boldsymbol{E}$ is applied at $100 \mathrm{~Hz} . P, A, \boldsymbol{E}$, and $R$ denote polarizer, analyzer, electric field, and rubbing directions.

rotation around the long axis is faster by a factor of at least 100, there has thus been an increasing interest in the exploitation of biaxial nematics in fast displays as well their use in stabilizing the blue phases. In smectics, the switching in $\operatorname{Sm} A P_{R}$ and $\operatorname{Sm} A P_{A}$ phases is brought by $E$ interacting with the dipole moment directed along the minor director. There is a considerable advantage to realizing switching in the smectic phases since the major director is already fixed by the smectic layers unlike in biaxial nematics where it needs to be anchored independently of the secondary director by external forces $[16,17]$. The switching speed in smectics may also be higher since $E$ interacts with macroscopic polarization as opposed to dielectric biaxiality in biaxial nematics.

In this letter, we determine the biaxiality and electricfield-induced biaxialities in $\operatorname{Sm} A P_{R}$ and $\operatorname{Sm} A P_{A}$ phases for the first time. We find that in the $\operatorname{Sm} A P_{A}$ phase, the structure transforms from one biaxial into another biaxial via a quasi-stable uniaxial structure upon the application of electric field. We also observe a large second harmonic electro-optic response in the $\operatorname{Sm} A P_{A}$ phase. The compound investigated is a 4-cyanoresorcinol bisbenzoate with two terephthalate-based wings (CK64), synthesized in Halle, Germany, whose molecular structure, phase sequence and phase transition temperatures (on cooling, in ${ }^{\circ} \mathrm{C}$ ) are shown in fig. 1 . We find that this material is the first compound where three different variants of the orthogonal $\operatorname{Sm} A$ phase $\left(\operatorname{Sm} A P_{A^{-}}-\operatorname{Sm} A P_{R^{-}} \operatorname{Sm} A\right)$ exist and both $\operatorname{Sm} A P_{A}-\operatorname{Sm} A P_{R}$ phases occur over a much broader temperature range and it is also the first example for a phase sequence where the latter occurs in between the two $\operatorname{Sm} A$ phases: uniaxial $\operatorname{Sm} A$ and biaxial $\operatorname{Sm} A P_{A}$ phases [18]. In the macroscopically uniaxial $\operatorname{Sm} A P_{R}$ phase, the primary director is orthogonal to the smectic layer plane. The secondary director consisting of short molecular axes coincides with the polar director, leading to an in-plane polarization. The direction of this polarization, however, is randomly distributed between the smectic layers $[13,14]$ or the magnitude of the azimuthal phase difference between the adjacent layers is constant 
but its sign varies randomly from one neighboring layer to another, resulting in a random and macroscopically uniaxial structure [13].

The textural changes under crossed polarizers of this sample in both homeotropic and planar cells as functions of temperature and applied electric field are given in fig. 1. A polymer (JSR Co.) is used for homeotropic alignment. Two electrode stripes are formed by etching indium tin oxide (ITO) on the bottom glass plate. An in-plane $E$ of frequency $100 \mathrm{~Hz}$ is applied between the ITO electrodes such that $E$ makes an angle of $45^{\circ}$ to the polarizer. The distance between these electrodes is fixed to be $\sim 200 \mu \mathrm{m}$. Values of birefringence and biaxiality were measured using a tilting optical compensator. This technique allows for the measurements of an extremely small optical retardation. This also allows us to measure the birefringence of a particular area (domain) in the cell even having a non-uniform texture such as the schlieren texture, etc. Strictly speaking the "birefringence" is applicable to the uniaxial structure such as $n_{e}-n_{o}$. For the biaxial structure there are three distinct eigenvalues $n_{X}, n_{Y}$ and $n_{Z}$. Therefore the smallest difference in refractive indices, $n_{Y}-n_{X}$, is the "biaxiality" and the difference between the others two is the "birefringence". Consequently, the term "birefringence" is used for planar cells while "biaxiality" is used for homeotropic ones. On cooling the sample in a homeotropic aligned cell in the absence of $E$ from the isotropic to $\operatorname{Sm} A$ and then to $\operatorname{Sm} A P_{R}$ phases, the cell shows very large extinction and a change in the direction of the polarization of light does not affect this extinction. The smectic layers in this arrangement lie parallel to the substrate (fig. 1(a)). We note that the application of in-plane $E$ produces no significant effect on the texture in the $\operatorname{Sm} A$ phase, but induces biaxiality in $\mathrm{Sm} A P_{R}$ as shown in fig. 1(b) where the secondary directors are directed parallel to $E$. This effect becomes stronger on approaching the phase transition to the $\operatorname{Sm} A P_{A}$ phase. The cell shows fast and reversible electro-optical switching from the field-induced bright to the dark state after the field is removed. On a further cooling to $111^{\circ} \mathrm{C}$ in the absence of $E$, the sample shows a phase transition to the $\mathrm{Sm}_{A} A P_{A}$ phase, characterized by the presence of a typical schlieren texture (fig. 1(c)), indicating the biaxial nature of this phase. On the application of $E$, textures show several, unexpected and interesting transformations (fig. 1(c)-(f)). Initially for a rather small $E(\sim 0.35 \mathrm{~V} / \mu \mathrm{m})$ the schlieren texture transforms into a uniform pattern (fig. 1(d)) with biaxiality of $\delta n \sim 0.016$ and the optical axis (i.e. secondary director $\boldsymbol{m}$ ) is directed along the electric field. An increase in $E$ causes an unexpected sharp drop in the macroscopic biaxiality to zero $(\delta n=0$, fig. 1(e)) for a critical electric field $E_{C}(T)$ which increases on cooling. An additional small increase in $E$, causes a rapid change in the uniaxial texture to the texture with biaxiality as before $\delta n=0.016$ (fig. $1(\mathrm{f})$ ). The biaxiality remains saturated with a further increase in $E$. The initial (fig. 1(d)) and final (fig. 1(f)) textures have the same biaxiality and these are optically identical to each other.

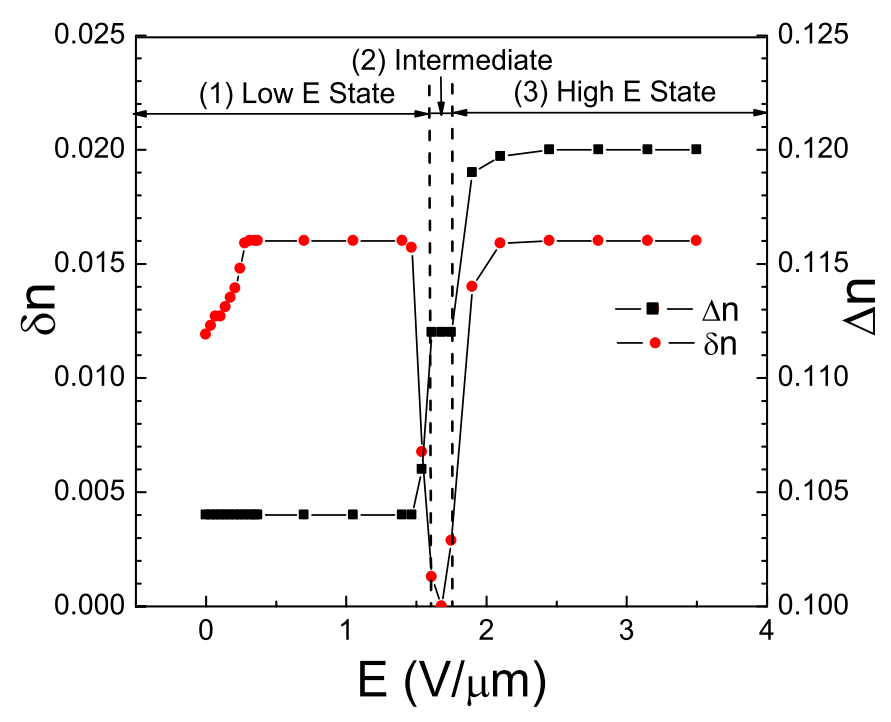

Fig. 2: (Colour on-line) Electric-field dependence of the biaxiality $\delta n(\square)$ and birefringence $\Delta n(\bullet)$ at $95^{\circ} \mathrm{C}$ measured in the $\mathrm{Sm}_{A} P_{A}$ phase in $8.7 \mu \mathrm{m}$ homeotropic and $9.5 \mu \mathrm{m}$ planar cells, respectively; the frequency of $E$ is $100 \mathrm{~Hz}$.

Similar transformations are also observed in a planar cell as well. Planar cells are prepared from low-resistance $(<20 \Omega / \square)$ ITO-coated glasses and polymer stripes are used as spacers. To achieve homogeneous alignment both substrates were coated with the RN1175 (Nissan Chemical Co. Japan) polymer and rubbed. On cooling from the isotropic to $\operatorname{Sm} A$ and then to $\operatorname{Sm} A P_{R}$ phases in the absence of $E$, the cell shows well-aligned planar texture with the primary director (major optical axis) lying along the rubbing direction. The quality of alignment is significantly improved by the application of a large ac electric field of variable frequency (fig. $1(\mathrm{~g})$, inset) across the cell. The uniform texture of large extinction is obtained with the cell in between the crossed polarizers such that the rubbing direction lies along the polarizer. In the absence of $E$ when the angle between the rubbing direction and the polarizer axis is $45^{\circ}$, the cell exhibits yellow color with a birefringence of $\Delta n \sim 0.104$ (fig. $1(\mathrm{~g})$ ). For a planar cell, the application of $E$ below $1.4 \mathrm{~V} / \mu \mathrm{m}$ produces no effect on the birefringence. On increasing the field from $1.5 \mathrm{~V} / \mu \mathrm{m}$ to $1.6 \mathrm{~V} / \mu \mathrm{m}$, the textural color changes from yellow to orange (fig. $1(\mathrm{~h})$, central area) with $\Delta n \sim 0.112$, seen through the microscope separated by a sharp domain wall. Note that this is the value of $E$ for which the homeotropic cell becomes uniaxial (fig. 1(e)). Again for $E$ of $1.8 \mathrm{~V} / \mu \mathrm{m}$, the cell changes its color to red with an effective birefringence of $\Delta n \sim 0.12$. Again the area of the cell observed through the microscope's aperture has a sharp domain boundary. For a certain field three areas with different birefringences can be seen in the micrograph (fig. 1(h)). These areas with different values of birefringence exist due to a slight temperature gradient in the cell. Results of measurements on biaxiality/birefringence for both homeotropic/planar cells in the $\operatorname{Sm} A P_{A}$ phase 


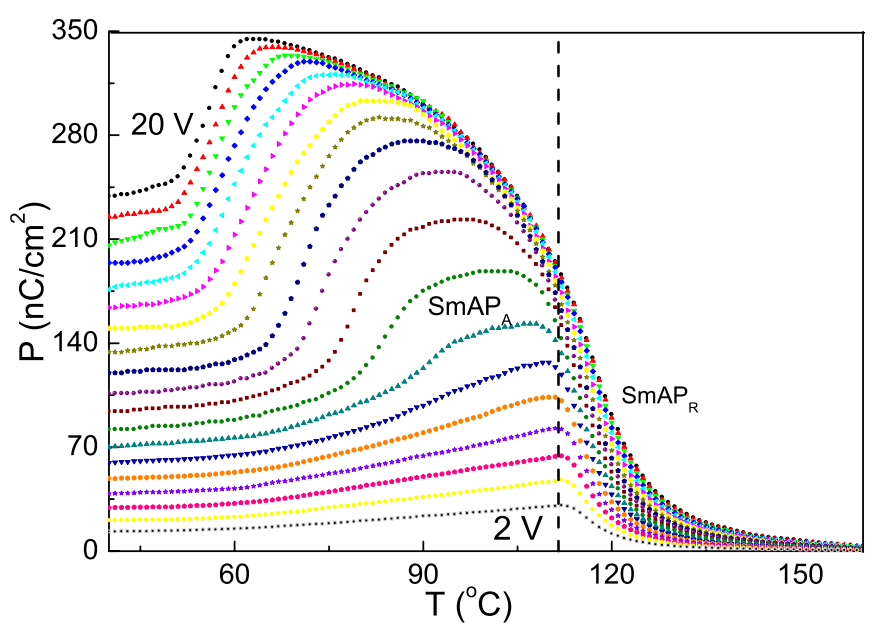

Fig. 3: (Colour on-line) Temperature dependence of the polarization measured in a $2 \mu \mathrm{m}$ planar cell for different applied voltages, from $2 \mathrm{~V}$ to $20 \mathrm{~V}, 100 \mathrm{~Hz}$ square wave with a step of $1 \mathrm{~V}$.

are summarized in fig. 2. Three important features are being noted: i) a difference in the birefringence value $(\Delta n)$ for high and low fields in a planar cell is equal to the biaxiality $(\delta n)$ in a homeotropic cell; ii) for an intermediate $E$ in the range $(1.6-1.8 \mathrm{~V} / \mu \mathrm{m})$, the planar cell has an intermediate birefringence value, while biaxiality of the homeotropic cell drops to zero and iii) a difference in the birefringence values in the planar cell in between the intermediate and low/high field states is equal to one-half the value of the biaxiality in a homeotropic cell. The inter-relations between the measured birefringence values can be written as follows: $\Delta n_{H}-\Delta n_{L} \approx \delta n_{H} \approx$ $\delta n_{L} \approx 0.016$ and $\Delta n_{H}-\Delta n_{I} \approx \Delta n_{I}-\Delta n_{L} \approx \delta n_{L} / 2 \approx$ 0.008 , where subscripts $L, H$ and $I$ denote low, intermediate and high electric fields, respectively.

Before determining the physical mechanisms involved in such textural transformations, we present results of the net polarization, $P$ (fig. 3). The important feature of this sample is the absence of $P$ in the entire temperature range when the field applied for its measurement is low. The application of $E$ above a threshold changes the distribution of the dipole moments (polar directors) and consequently gives rise to the net polarization $P$. Figure 3 shows the temperature dependence of the measured polarization in a planar cell of thickness $2 \mu \mathrm{m}$ for both $\operatorname{Sm} A P_{A}$ and $\operatorname{Sm} A P_{R}$ phases. The polarization is measured using the integral current technique. In the higher temperature range of the $\operatorname{Sm} A P_{R}$ phase, we find that the induced polarization is linearly proportional to the applied field, which increases on cooling down to the $\operatorname{Sm} A P_{A}$ phase. It is observed that $P(T)$ increases on approaching the $\operatorname{Sm} A P_{R^{-}}$ $\mathrm{Sm} A P_{A}$ phase transition. In the temperature range corresponding to the $\operatorname{Sm}_{A} A P_{A}$ phase, the net polarization is initially proportional to the applied field and then it jumps to a saturated value. The decrease in the polarization at lower temperatures is for the reason that much higher voltage is needed to saturate it. It may be remarked that while the field-induced polarization shows a smooth-like normal dependence on $E$, the biaxiality (fig. 2) shows a sharp and step-like behavior. This arises from the principal difference of the manner used in arriving at the results using the two techniques. The birefringence is measured for a localized small area in the cell, whereas the polarization is measured over the entire area of the cell. The latter may consist of three different domains shown in fig. 1(h) and hence the measured $P$ corresponds to an integrated response over the entire area.

The suggested switching mechanisms in the sample under electric field are presented in fig. 4(a). In the $\operatorname{Sm} A P_{R}$ phase and in the absence of $E$ across the cell, the dipoles and therefore the polar directors are randomly distributed as found in the literature $[14,15]$. On applying an in-plane electric field, the dipoles gradually orient towards the field direction inducing macroscopic polarization and biaxiality; with the secondary optical axis of the polar directors in a homeotropic cell is directed along the applied field (fig. 1(b)). The distortion of the random distribution obeys the two-dimensional Langevin equation [15]. In the $\operatorname{Sm}_{A} P_{A}$ phase, the short molecular axes in single smectic layers are aligned similarly but anti-parallelly in the neighboring layers thus forming an antiferroelectric structure. The response of this structure to the electric field is no longer continuous (Langevin-like) but shows three optically distinguishable states (fig. 1(h) and fig. 2). The application of a rather small electric field slightly distorts the initial antiferroelectric structure as shown in fig. 4(a) so that it gives rise to the measurable polarization. For higher fields, this initial antiferroelectric structure (fig. 1(d)) completely transforms into a ferroelectric (fig. 1(f)) structure. In the very narrow range of intermediate $E$, the homeotropic cell shows almost zero birefringence (fig. 1(e)) arising from zero biaxiality; this is only possible when the angle between the local polarizations vectors (and therefore the polar directors) is $90^{\circ}$ (fig. 4(a)). This state in the planar cell exhibits an intermediate birefringence value of 0.112 , which lies exactly in between the birefringence values in the antiferroelectric and ferroelectric states (fig. 1(h)).

It is possible to estimate the birefringence as well as the biaxiality in different states for different alignment conditions. First, we introduce the biaxiality model of the orthogonal smectic layer formed by bent-shape molecules by two optically equivalent anticlinically tilted layers formed by rod-shaped molecules (i.e. fragments of bentshaped molecules) with refractive indices $n_{e}$ (along the long fragment's axis) and $n_{o}$, in each layer and with the tilt angle related to the opening angle $(\psi)$ of the bent-shape molecules as shown in fig. 4(b), (c). This forms anisotropic bi-layered media with three distinct eigenvalues that can be labeled as refractive indices: $n_{Z}$ - along the smectic layer nomal (primary molecular director $\vec{n}$ ), $n_{Y}$ - along the tilt direction (the secondary molecular director $\vec{m}$ ), and $n_{X}$ - which is perpendicular to the previous two eigenvalues. Refractive indices 


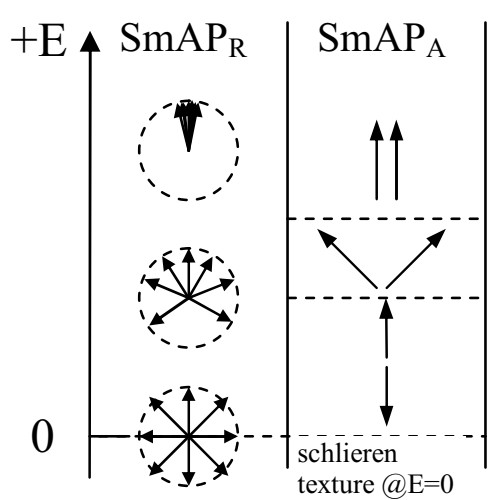

(a)

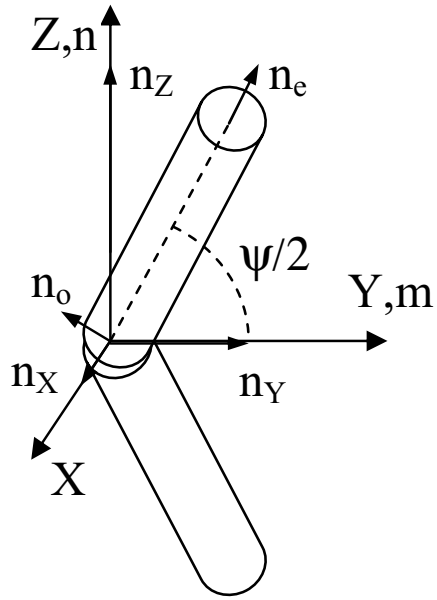

(b)

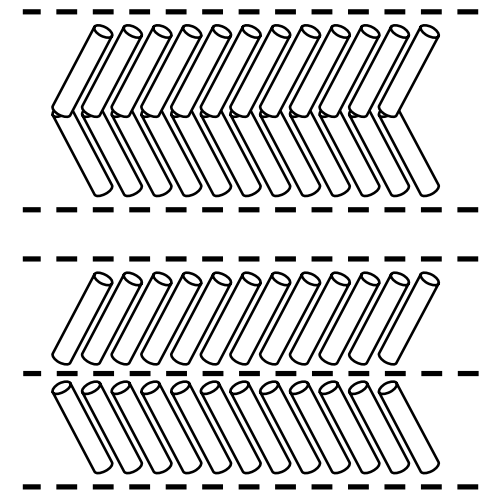

(c)

Fig. 4: (a) Ordering and switching mechanisms in $\operatorname{Sm} A P_{R}$ and $\operatorname{Sm} A P_{A}$ phases with increase in field $E$ in the vertical direction. The arrows represent the secondary molecular axes and local polarization vectors; (b) the biaxiality model for bent-core molecule. $Z$ and $Y$ axes lie along the primary, $\boldsymbol{n}$, and secondary, $\boldsymbol{m}$, directors; (c) Model representation of one orthogonal bent-core smectic layer (upper diagram) by two optically equivalent tilted layers (lower diagram).

$n_{X}, n_{Y}$ and $n_{Z}$ can be estimated using the well-known formula: $n(\theta)=n_{o} n_{e} /\left[\sqrt{n_{e}^{2} \sin ^{2}(\theta)+n_{o}^{2} \cos ^{2}(\theta)}\right]$. Here $\theta$ is an angle between the polarization of light and the optical axis, $n_{e}$ and $n_{o}$ are the refractive indices of the single layer, along and normal to the major axis. For the model proposed (fig. 4(b)): $n_{Z}=n(90-\psi / 2), n_{Y}=$ $n(\psi / 2), n_{X}=n_{o}$. Using this model, we find three different refractive indices values $\Delta n_{Z X}=n_{Z}-n_{X} ; \Delta n_{Z Y}=n_{Z}-$ $n_{Y} ; \Delta n_{Y X}=n_{Y}-n_{X} ;$ where $\Delta n_{Z X}-\Delta n_{Z Y}=\Delta n_{Y X}$, the latter turns out to be the biaxiality $\left(\Delta n_{Y X}=\delta n\right)$. In the homeotropic cell, both antiferroelectric and ferroelectric structures must have the same value of biaxiality, equal to $\delta n=0.016$ (see fig. 1(d), (f) and fig. 2). For the planar cell in the absence or under low $E$, the molecular planes are parallel to the surface and the birefringence must be equal to $\Delta n_{L}=\Delta n_{Z Y}=0.104$. Under the high electric field the molecular planes are perpendicular to the surface and the macroscopic birefringence must equal to $\Delta n_{H}=\Delta n_{Z X}=0.12$. According to the model proposed here, all three refractive indices depend on only two independent parameters: $n_{e}-n_{o}, \psi$. By fitting this equation to the experimental data, these are found to be: $n_{e}-n_{o}=$ 0.14 and $\psi=138^{\circ}$. The opening angle, $\psi=138^{\circ}$, obtained from fitting the data lies in the range $135-140^{\circ}$ [19] and this agrees with that found by NMR experiments for systems similar to CK64. Also note that for intermediate $E$, the macroscopic biaxiality in the homeotropic cell is observed to be zero and in the planar cell the birefringence is found to be 0.112 (fig. 2). Finally, let us examine the possible application based on the electro-optic effect in a homeotropic cell to displays. The feasibility of the electro-optic effect in $\operatorname{Sm} A P_{R}$ was studied in [14] showing several advantages over existing LCD modes, such as the fast response $(100 \mu \mathrm{s})$, high contrast ratio (3000: 1), wide viewing angle and continuous gray scale. Nevertheless, it requires a rather large field $E$ (up to $10 \mathrm{~V} / \mu \mathrm{m}$ ) which for the homeotropic sample may reach $100 \mathrm{~V}$ or more. This voltage is also strongly temperature dependent (fig. 3). In contrast to $\operatorname{Sm} A P_{R}$, the electro-optic effect in $\operatorname{Sm} A P_{A}$ requires much smaller applied voltage. The high contrast electro-optic switching can be achieved between the intermediate dark (fig. 1(e)) and bright high/low-voltage states (fig. $1(\mathrm{~d}) /(\mathrm{f}))$. The feasibility study of this effect is in progress.

In conclusion, the structural and electro-optical phenomena are investigated in an achiral biaxial bentcore molecular compound with $\operatorname{Sm} A P_{A}$ and $\operatorname{Sm} A P_{R}$ phases. The homeotropic cell in the $\operatorname{Sm} A P_{A}$ phase shows an unusual transition from an initially biaxial texture to a quasi-stable uniaxial one and then again to a biaxial structure upon the application of electric field. The last state (high $E$ ) has the same biaxiality as the initial one (low $E$ ) with high and low fields, respectively. These transformations are explained with successive transitions from antiferroelectric to ferroelectric states. In the intermediate state, a uniaxial structure is formed where the secondary directors in the neighboring smectic layers are perpendicular to each other. The biaxiality/birefringence calculated with the model for the bent-core molecules shows a good quantitative agreement with those obtained experimentally.

We acknowledge funding by EU FP7-216025 BIND and SFI RFP 06/RFP/ENE039 projects. 


\section{REFERENCES}

[1] Meyer R. B., Liebert L., Strzelecki L. and Keller P., J. Phys. (Paris), 36 (1975) L69.

[2] Brand H. R., Cladis P. E. and Pleiner H., Macromolecules, 25 (1992) 7223.

[3] Niori T., Sekine T., Watanabe J., Furukawa T. and Takezoe H., J. Mater. Chem., 6 (1996) 1231.

[4] Link D. R., Natale G., Shao R., Maclennan J. E., Clark N. A., Korblova E. and Walba D. M., Science, 278 (1997) 1924.

[5] Kruerke D. and Heppke G., presented at the Gordon Conference on Liquid Crystals, 1997, New Hampshire; Banana-shaped Liquid Crystal Workshop, 1997, Berlin, Germany.

[6] Sekine T., Niori T., Watanabe J., Furukawa T., Choi S. W. and Takezoe H., J. Mater. Chem., 7 (1997) 1307; Sekine T., Niori T., Sone M., Watanabe J., Choi S. W., Takanishi Y. and Takezoe H., Jpn. J. Appl. Phys., 36 (1997) 6455.

[7] Pelzl G., Diele S. and Weissflog W., Adv. Mater., 11 (1999) 707.

[8] Reddy A. R. and Tschierske C., J. Mater. Chem., 16 (2006) 907961.

[9] Ros M. B., Serrano J. L., De la Fuente M. R. and Folcia C. L., J. Mater. Chem., 15 (2005) 5093.
[10] Takezoe H. and Takanishi Y., Jpn. J. Appl. Phys., 45 (2006) 597.

[11] Eremin A., Diele S., Pelzl G., Nadasi H., Weissflog W., Salfetnikova J. and Kresse H., Phys. Rev. E, 64 (2001) 051707.

[12] Schroder M. W., Diele S., Pancenko N., Weissflog W. and Pelzl G., J. Mater. Chem., 12 (2002) 1331.

[13] Pociecha D., Cepic M., Gorecka E. and Mieczkowski J., Phys. Rev. Lett., 91 (2003) 185501.

[14] Shimbo Y., Takanishi Y., Ishikawa K., Gorecka E., Pociecha D., Mieczkowski J., Gomola K. and Takezoe H., Jpn. J. Appl. Phys., 45 (2006) L282.

[15] Shimbo Y., Gorecka E., Pociecha D., Araoka F., Goto M., Takanishi Y., Ishikawa K., Mieczkowski J., Gomola K. and Takezoe H., Phys. Rev. Lett., 97 (2006) 113901.

[16] Acharya B. R., Primak A. and Kumar S., Phys. Rev. Lett., 92 (2004) 145506.

[17] Nagaraj M., Panarin Y. P., Manna U., Vij J. K., Keith C. and Tschierske C., Appl. Phys. Lett., 96 (2010) 011106.

[18] Keith C., Prehm M., Panarin Y. P., Vij J. K. and Tschierske C., Chem. Commun., 46 (2010) 3702.

[19] Wirth I., Diele S., Eremin A., Pelzl G., Grande S., Kovalenko L., Panfenko N. and Weissflog W., J. Mater. Chem., 11 (2001) 1642. 